I.V. Bilynskyi, R.Ya. Leshko, H.O. Metsan, I.S. Shevchuk

\title{
Hole States in Spherical Quantum Nanoheterosystem with Intermediate Spin-Orbital Interaction
}

\author{
Drohobych Ivan Franko State Pedagogical University, 3 Stryiska Str., 82100 Drohobych, Ukraine, \\ email:leshkoroman@gmail.com
}

\begin{abstract}
The hole energy spectrum has been studied for the spherical semiconductor nanoheterosystem with the cubic symmetry. The exact solutions of the Schrödinger equation for the ground and excited hole states are presented within the framework of the 6-band Luttinger Hamiltonian and the finite gap of bands with the corresponding boundary conditions. Dependence of the holes energies from the radius of the quantum dot has been calculated for the GaAs/AlAs heterostructure. Obtained results where compared with data obtained using the infinite potential well model, as well as the single-band model for heavy and light holes.
\end{abstract}

Keywords: quantum don, hole spectrum, 6-band model.

Стаття поступила до редакиії 12.08.2019; прийнята до друку 15.09.2019.

\section{Introduction}

Significant success has been achieved in the theoretical and experimental studies of nanoheterostructures over the past two decades. They are the most interesting and promising for practical application. Heterostructures with quantum dots (QDs) are considered to be very perspective optical (laser environments, converters of radiation) and electric (field transistors, solar cells) materials. Optical and electrical properties of the QD (InAs, GaAs, CdS, CdTe, etc.) are widely used in photoelectric [2], photodynamic therapy $[3,4]$ and biology $[5,6]$. For example, a change in the effective band gap of the $C d S$ QD due to the size quantization effect covers the wavelength range from 300 to $520 \mathrm{~nm}$ in optical absorption and from 400 to $700 \mathrm{~nm}$ in luminescence [7].

In addition to the composition and size of a serious influence on the properties of QD provides their form. The spherical QD is the most simple to manufacture and currently have the greatest practical application. In addition, such structures are also of interest in terms of their physical properties, since current carriers (electrons, holes) are characterized by confinement of their motion in all three dimensions. Therefore, there are many theoretical publications concerning the investigation of the spectra of electrons, holes and excitons in quantum dots of different configurations [8-10].

The discrete structure of the QDs energy spectrum is manifested primarily in the absorption spectra [2]. Peaks are clearly visible in these spectra in the case of a small change in the QD size. These peaks correspond to interband optical transitions that are related with different electron and hole quantum states. These features can be described within the framework of the effective mass model.

In the study of electronic states often the complex structure of the valence band in semiconductors, that forming the heterosystem $[9,11-12,15,16]$, don't take into account. In these cases, the effective mass approximation were used. The inhomogeneous change in the effective mass of quasiparticles near heterojunctions is also taken into account, the so-called single-band approximation. These approximations are suitable for electronic states of wide-band crystals forming a heterosystem.

For hole states, this approximation gives inaccurate results in energy and it is unable to describe a series of optical characteristics. As shown in [13], the first two peaks in absorption spectra are related to transitions between the lower electron (1S) and two different hole levels $\left(1 \mathrm{~S}_{3 / 2}\right.$ and $\left.2 \mathrm{~S}_{3 / 2}\right)$. In the QDs spectra with a radius of $4.1 \mathrm{~nm}$, the bands corresponding to the transition between the $1 \mathrm{~S}$ electronic state and the hole level are also observed, resulting from the spin-orbit splitting $\left(3 \mathrm{~S}_{1 / 2}\right)$. That is why multiband approximations should be used for the correct description. In the series of theoretical works [14-16] for single-layer QD heterosystems $\mathrm{GaSb} / \mathrm{AlSb}$ and for double-layer CdSe/ZnSe/vacuum where calculated the electron and hole energy states and the absorption coefficient at different QD radius. In this 
case, the parameters of these systems for hole states allow the use of a $4 \times 4$ band model, since for a massive $G a S b$ crystal the value of the spin-orbital hole band is large $(\Delta=0.77 \mathrm{eV})$ and for $C d T e \Delta=0.91 \mathrm{eV}$, so its influence can be neglected. However, for the heterosystems $\quad \operatorname{GaAs}(\Delta=0.34) / A l A s(\Delta=0.28)$, $\operatorname{InAs}(\Delta=0.38) / G a A s(\Delta=0.34), \quad$ it is necessary to consider the light and heavy holes bands, as well as the spin-split band, that is, the so-called six-band Luttinger model. The complex structure of the valence band leads to the mixing of heavy and light hole states on the interfaces, which is caused in a qualitative change in their energy spectrum and wave functions.

The study of spin-splitting of the valence band allows us to determine the microscopic parameters that describe the hole energy spectrum, the geometric shape and size of the nanostructure, the value of the spin-orbital interaction, and also provides a new tool for studying the symmetry of nanobjects.

That is why the aim of this work are:

- the study of the influence of the complex structure of the valence band (bands of light and heavy holes and spin-split hole band) on the energy spectrum of nanoheterosytems;

- the study of the dependence of different hole states on the QD radius;

- the qualitative and quantitative compare obtained results for different models of hole states.

\section{Problem statement. basic formulas}

The spherical semiconductor nanocrystal with radius $R$, which is placed in a semiconductor matrix, is considered. The hole is confined in a spherically symmetric finite rectangular potential well due to the band offset. The potential energy is given by the expression:

$$
U=\left\{\begin{array}{l}
0, r<R \\
V_{0}, r>R
\end{array}\right.
$$

Let's consider that the QD material and the matrix are semiconductors with cubic symmetry. The value of the spin-orbital splitting in the valence band, and the Luttinger parameters $\gamma_{3}$ and $\gamma_{2}$ are approximately the same $[18,19]$.

The analysis is carried out using the kp-method [20], in the framework of the six-band Hamiltonian, which describes the energy spectrum of the valence band, neglecting the corrugation of isoenergy surfaces.

The atomic system of units is used in calculations for convenience. The Hamiltonian of the system in the matrix form is written in this way:

$$
H=\left|\begin{array}{cccccc}
P+Q & L & M & 0 & i \sqrt{1 / 2} L & -i \sqrt{2} M \\
L^{*} & P-Q & 0 & M & -i \sqrt{2} Q & i \sqrt{3 / 2} L \\
M^{*} & 0 & P-Q & -L & -i \sqrt{3 / 2} L^{*} & -i \sqrt{2} Q \\
0 & M^{*} & -L^{*} & P+Q & -i \sqrt{2} M^{*} & -i \sqrt{1 / 2} L^{*} \\
-i \sqrt{1 / 2} L^{*} & i \sqrt{2} Q & i \sqrt{3 / 2} L & i \sqrt{2} M & P-\Delta & 0 \\
i \sqrt{2} M^{*}-i \sqrt{3 / 2} L^{*} & i \sqrt{2} Q & i \sqrt{1 / 2} L & 0 & P-\Delta
\end{array}\right|+U
$$

where following notations are introduced

$$
\begin{gathered}
P=\frac{\gamma_{1}}{2} p^{2}, Q=\frac{\gamma}{2}\left(p_{\perp}^{2}-2 p_{z}^{2}\right), L=-i \sqrt{3} \gamma p-p_{z}, M=\sqrt{3 / 2} \gamma p_{z}^{2}, \\
p_{\perp}^{2}=p_{x}^{2}+p_{y}^{2}, p_{ \pm}^{2}=p_{x} \pm i p_{y}, p_{x}=-i \frac{\partial}{\partial x}, p_{y}=-i \frac{\partial}{\partial y}, p_{z}=-i \frac{\partial}{\partial z}
\end{gathered}
$$

$\gamma, \gamma_{1}$ - are the Luttinger parameters which set the effective masses of heavy and light holes: $m_{l}=1 /\left(\gamma_{1}+2 \gamma\right), m_{h}=\frac{1}{\left(\gamma_{1}-2 \gamma\right)}, p_{\mathrm{i}}$ is momentum

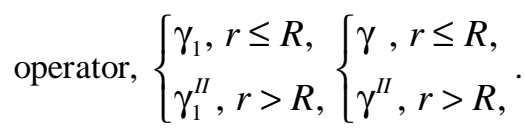

Particles moving in a spherically symmetric field. Its states are described by the stationary Schrödinger equation:

$$
H \psi=E \psi
$$

After substituting in (4) the spherically symmetric solutions obtained in [21] for even $\left(\psi_{j}^{+}\right)$and odd $\left(\psi_{j}^{-}\right)$ hole states with a full momentum $j$ 


$$
\begin{aligned}
& \psi_{j}^{+}=\left(\begin{array}{c}
\frac{R_{h 2}^{+}}{\sqrt{2 j(2 j-1)(2 j-2)}} \Phi_{j-3 / 2}^{(4)}+\frac{R_{h 1}^{+}}{\sqrt{2 j(2 j+2)(2 j+3)}} \Phi_{j+1 / 2}^{(4)} \\
R_{s}^{+} \Phi_{j+1 / 2}^{(2)}
\end{array}\right) \\
& \Psi_{j}^{-}=\left(\begin{array}{c}
\frac{R_{h 2}^{-}}{\sqrt{2(j+1)(2 j+3)(2 j+4)}} \Phi_{I+3 / 2}^{(4)}+\frac{R_{h 1}^{+}}{\sqrt{2 j(2 j-1)(2 j+2)}} \Phi_{j-1 / 2}^{(4)} \\
R_{s}^{-} \Phi_{j-1 / 2}^{(2)}
\end{array}\right),
\end{aligned}
$$

where $\Phi_{k}^{(4)}, \Phi_{k}^{(2)}$ are four-dimensional and two-dimensional vectors-columns [20] based on spherical harmonics $Y_{l, m}(\theta, \varphi)$. We obtain two systems of equations for the radial components of the holes eigenfunctions, $R_{h 1}, R_{h 2}, R_{S}$ are located in the QD and outside QD $\left(j=\frac{1}{2}, \frac{3}{2}, \frac{5}{2}, \ldots\right)$.

For even holes states in the QD system of differential equations will look like:

$$
\begin{aligned}
& -\left(\frac{\gamma_{1}}{2}-\frac{\gamma}{2} \frac{2 j-3}{2 j}\right)\left(\Delta_{j+1 / 2} R_{h 1}^{+}\right)-\frac{\gamma}{4 j} \sqrt{3(2 j-1)(2 j+3)}\left(A_{j-3 / 2}^{+} R_{h 2}^{+}\right)-\frac{\gamma}{2} \sqrt{\frac{2 j+3}{j}}\left(\Delta_{j+1 / 2} R_{S}^{+}\right)-E R_{h 1}^{+}=0, \\
& -\left(\frac{\gamma_{1}}{2}+\frac{\gamma}{2} \frac{2 j-3}{2 j}\right)\left(\Delta_{j-3 / 2} R_{h 2}^{+}\right)-\frac{\gamma}{4 j} \sqrt{3(2 j-1)(2 j+3)}\left(A_{j+1 / 2}^{-} R_{h 1}^{+}\right)-\frac{\gamma}{2} \sqrt{\frac{3(2 j-1)}{j}}\left(A_{j+1 / 2}^{-} R_{s}^{+}\right)-E R_{h 2}^{+}=0, \\
& -\frac{\gamma}{2} \sqrt{\frac{2 j+3}{j}}\left(\Delta_{j+1 / 2} R_{h 1}^{+}\right)-\frac{\gamma}{2} \sqrt{\frac{3(2 j-1)}{j}}\left(A_{j-3 / 2}^{+} R_{h 2}^{+}\right)-\frac{\gamma_{1}}{2}\left(\Delta_{j+1 / 2} R_{s}^{+}\right)-(E-\Delta) R_{s}^{+}=0,
\end{aligned}
$$

and for odd states

$$
\begin{aligned}
& -\left(\frac{\gamma_{1}}{2}-\frac{\gamma}{2} \frac{2 j+5}{(j+1)}\right)\left(\Delta_{j-1 / 2} R_{h 1}^{-}\right)+\frac{\gamma}{4(j+1)} \sqrt{3(2 j-1)(2 j+3)}\left(A_{j+3 / 2}^{-} R_{h 2}^{-}\right)+\frac{\gamma}{2} \sqrt{\frac{2 j-1}{j+1}}\left(\Delta_{j-1 / 2} R_{S}^{-}\right)-E R_{h 1}^{-}=0, \\
& -\left(\frac{\gamma_{1}}{2}+\frac{\gamma}{2} \frac{2 j+5}{2(j+1)}\right)\left(\Delta_{j+3 / 2} R_{h 2}^{-}\right)+\frac{\gamma}{4(j+1)} \sqrt{3(2 j-1)(2 j+3)}\left(A_{j-1 / 2}^{+} R_{h 1}^{-}\right)-\frac{\gamma}{2} \sqrt{\frac{3(2 j+3)}{j+1}}\left(A_{j-1 / 2}^{+} R_{s}^{-}\right)-E R_{h 2}^{-}=0, \\
& \frac{\gamma}{2} \sqrt{\frac{2 j-1}{j+1}}\left(\Delta_{j-1 / 2} R_{h 1}^{-}\right)-\frac{\gamma}{2} \sqrt{\frac{3(2 j+3)}{j+1}}\left(A_{j+3 / 2}^{-} R_{h 2}^{-}\right)-\frac{\gamma_{1}}{2}\left(\Delta_{j-1 / 2} R_{s}^{-}\right)-(E-\Delta) R_{s}^{-}=0,
\end{aligned}
$$

where $E, \Delta$ are dimensionless quantities and the following notations are introduced:

$$
\Delta_{l}=\frac{\partial^{2}}{\partial r^{2}}+\frac{2}{r} \frac{\partial}{\partial r}-\frac{l(l+1)}{r^{2}}, A_{l}^{-}=\frac{\partial^{2}}{\partial r^{2}}+\frac{2 l+1}{r} \frac{\partial}{\partial r}-\frac{l^{2}-1}{r^{2}}, A_{l}^{+}=\frac{\partial^{2}}{\partial r^{2}}-\frac{2 l-1}{r} \frac{\partial}{\partial r}+\frac{l(l-2)}{r^{2}} .
$$

Systems of differential equations will have exact solutions for even and odd states. In the inner region for a spherical QD, the solutions of the equations system (radial functions), are written using the sum of the three spherical Bessel functions of the first kind:

$$
\begin{aligned}
& R_{h 1}^{j+}(r)=c_{1} J_{j+1 / 2}\left(k_{l} r\right)+c_{2} J_{j+1 / 2}\left(k_{h} r\right)+c_{3} J_{j+1 / 2}\left(k_{s} r\right), \\
& R_{h 2}^{j+}(r)=-c_{1} \sqrt{\frac{3(2 j-1)}{2 j+3}} J_{j-3 / 2}\left(k_{l} r\right)+c_{2} \sqrt{\frac{2 j+3)}{3(2 j-1)}} J_{j-3 / 2}\left(k_{h} r\right)-c_{3} \sqrt{\frac{3(2 j-1)}{2 j+3}} J_{j+3 / 2}\left(k_{s} r\right), \\
& R_{s}^{j+}(r)=c_{1} \sqrt{\frac{j}{2 j+3}} \frac{2 E-\left(\gamma_{1}+2 \gamma\right) k_{l}^{2}}{\gamma k_{l}^{2}} J_{j+1 / 2}\left(k_{l} r\right)+c_{3} \sqrt{\frac{j}{2 j+3}} \frac{2 E-\left(\gamma_{1}+2 \gamma\right) k_{s}^{2}}{\gamma k_{s}^{2}} J_{j+1 / 2}\left(k_{s} r\right),
\end{aligned}
$$

and solutions for odd states

$$
\begin{aligned}
& R_{h 1}^{j-}(r)=c_{4} \sqrt{2 j-1} J_{j-1 / 2}\left(k_{l} r\right)+c_{5} \sqrt{2 j-1} J_{j-1 / 2}\left(k_{h} r\right)+c_{6} \sqrt{2 j-1} J_{j-1 / 2}\left(k_{s} r\right), \\
& R_{h 2}^{j-}(r)=c_{4} \sqrt{3(2 j+3)} J_{j+3 / 2}\left(k_{l} r\right)-c_{5} \frac{\sqrt{2 j-1}}{\sqrt{3(2 j+3)}} J_{j+3 / 2}\left(k_{h} r\right)+c_{6} \sqrt{3(2 j+3)} J_{j+3 / 2}\left(k_{s} r\right), \\
& R_{s}^{j-}(r)=c_{4} \sqrt{j+1} \frac{\left(\gamma_{1}+2 \gamma\right) k_{l}^{2}-2 E}{\gamma k_{l}^{2}} J_{j-1 / 2}\left(k_{l} r\right)+c_{6} \sqrt{j+1} \frac{\left(\gamma_{1}+2 \gamma\right) k_{s}^{2}-2 E}{\gamma k_{s}^{2}} J_{j-1 / 2}\left(k_{s} r\right) .
\end{aligned}
$$

The squares of the wave vectors $k_{h}, k_{l}, k_{s}$ are connected with the proper energy $E$ of the investigated hole 
states by the following relation:

$$
k_{h}^{2}=\frac{2 E}{\left(\gamma_{1}-2 \gamma\right)}, k_{l, s}^{2}=\frac{2 E\left(\gamma_{1}+\gamma\right)-\Delta\left(\gamma_{1}+2 \gamma\right) \pm \sqrt{\left[2 E\left(\gamma_{1}+\gamma\right)-\Delta\left(\gamma_{1}+2 \gamma\right)\right]^{2}-4 E(E-\Delta)\left(\gamma_{1}-2 \gamma\right)\left(\gamma_{1}+4 \gamma\right)}}{\left(\gamma_{1}-2 \gamma\right)\left(\gamma_{1}+4 \gamma\right)}
$$

In the matrix ( $r>R$ ), the solutions of the equations can be represented using modified spherical Bessel functions of the second kind for even (12) and odd states (13):

$$
\begin{aligned}
& R_{h 1}^{j+}(r)=c_{1} K_{j+1 / 2}\left(k_{l} r\right)+c_{2} K_{j+1 / 2}\left(k_{h} r\right)+c_{3} K_{j+1 / 2}\left(k_{s} r\right) \\
& R_{h 2}^{j+}(r)=-c_{1} \sqrt{\frac{3(2 j-1)}{2 j+3}} K_{j-3 / 2}\left(k_{l} r\right)+c_{2} \sqrt{\frac{2 j+3)}{3(2 j-1)}} K_{j-3 / 2}\left(k_{h} r\right)-c_{3} \sqrt{\frac{3(2 j-1)}{2 j+3}} K_{j+3 / 2}\left(k_{s} r\right), \\
& R_{s}^{j+}(r)=c_{1} \sqrt{\frac{j}{2 j+3}} \frac{2 \varepsilon-\left(\gamma_{1}+2 \gamma\right) k_{l}^{2}}{\gamma k_{l}^{2}} K_{j+1 / 2}\left(k_{l} r\right)+c_{3} \sqrt{\frac{j}{2 j+3}} \frac{2 \varepsilon-\left(\gamma_{1}+2 \gamma\right) k_{s}^{2}}{\gamma k_{s}^{2}} K_{j+1 / 2}\left(k_{s} r\right), \\
& R_{h 1}^{j-}(r)=c_{4} \sqrt{2 j-1} K_{j-1 / 2}\left(k_{l} r\right)+c_{5} \sqrt{2 j-1} K_{j-1 / 2}\left(k_{h} r\right)+c_{6} \sqrt{2 j-1} K_{j-1 / 2}\left(k_{s} r\right), \\
& R_{h 2}^{j-}(r)=c_{4} \sqrt{3(2 j+3)} K_{j+3 / 2}\left(k_{l} r\right)-c_{5} \frac{\sqrt{2 j-1}}{\sqrt{3(2 j+3)}} K_{j+3 / 2}\left(k_{h} r\right)+c_{6} \sqrt{3(2 j+3)} K_{j+3 / 2}\left(k_{s} r\right), \\
& R_{s}^{j-}(r)=c_{4} \sqrt{j+1} \frac{\left(\gamma_{1}+2 \gamma\right) k_{l}^{2}-2 E}{\gamma k_{l}^{2}} K_{j-1 / 2}\left(k_{l} r\right)+c_{6} \sqrt{j+1} \frac{\left(\gamma_{1}+2 \gamma\right) k_{s}^{2}-2 E}{\gamma k_{s}^{2}} K_{j-1 / 2}\left(k_{s} r\right)
\end{aligned}
$$

The squares of wave vectors $k_{l}, k_{h}, k_{s}$ are obtained from the formula (11) by substitution $E \rightarrow E-V_{0} \gamma_{1} \rightarrow \gamma_{1}^{I I}, \gamma \rightarrow \gamma^{I I}, \Delta \rightarrow \Delta^{I I}$.

If consider multiband models for planar heterostructure, than the boundary conditions for the wave function are obtained by integrating the Schrödinger equation through the heterointerfaces and taking into account the continuity of the envelope wave function [22]. The resulting boundary conditions are given below:

$$
\left.\psi_{A}\right|_{z=-0}=\left.\psi_{A}\right|_{z=+0},\left.\quad J_{z} \psi_{A}\right|_{z=-0}=\left.J_{z} \psi_{B}\right|_{z=+0},
$$

where $\mathrm{A}$ and $\mathrm{B}$ are two materials separated by a heterointerfaces $z=0$.

As in the case of flat heterostructures, for a spherical quantum dot, the Schrödinger radial equation has the following form

$$
\left(H_{j}^{(p)}-E_{j}^{(p)}\right) R_{j}^{(p)}=0, \quad R_{j}^{(p)}=\left(\begin{array}{c}
R_{h_{1}}^{j+} \\
R_{h_{2}}^{j+} \\
R_{h_{3}}^{j+}
\end{array}\right)
$$

where $E_{j}^{(p)}$ is the eigenenergies.

The Schrödinger equation is integrated through the points $r=R$ to obtain the boundary conditions. Where $r=R$ is spherical heterointerface that separates two materials. Taking into account the continuity of the radial wave function, the necessary boundary conditions have form:

$$
\begin{gathered}
\left.\left(R_{j}^{(p)}\right)_{A}\right|_{r=a-0}=\left.\left(R_{j}^{(p)}\right)_{B}\right|_{r=a+0}, \\
\left.J_{j}^{(p)}\left(R_{j}^{(p)}\right)_{A}\right|_{r=R-0}=\left.J_{j}^{(p)}\left(R_{j}^{(p)}\right)_{B}\right|_{r=R+0}
\end{gathered}
$$

The radial component of the matrix operator of the probability density flux is obtained from a radial Hamiltonian $H_{j}^{(p)}$. We take into account that the Hamiltonian contains an operator $A_{l}^{(p)}$, and for $\mathrm{p}=1$ the even states and $\mathrm{p}=-1$ are respectively odd states. We will get:

$$
J_{j}^{(p)}=\left(\begin{array}{ccc}
\gamma_{1} \frac{\partial}{\partial r}-c_{j}^{p} \gamma D_{r}+f_{-8}^{j, p} \frac{\underline{\chi}}{r} & p a_{j}^{p} b_{j}^{p} \gamma A_{j-3 p / 2}^{(p)} & p \sqrt{2} a_{j}^{p}\left(\gamma D_{r}+f_{1}^{j, p} \frac{\chi}{r}\right) \\
-p a_{j}^{p} b_{j}^{p} \gamma A_{j+p / 2}^{(-p)} & \gamma_{1} \frac{\partial}{\partial r}+c_{j}^{p} \gamma D_{r}-3 f_{4}^{j, p} \frac{\underline{\chi}}{r} & -\sqrt{2} a_{j}^{p} \gamma A_{j+p / 2}^{(-p)} \\
p \sqrt{2} a_{j}^{p}\left(\gamma D_{r}+f_{1}^{j, p} \frac{\underline{\chi}}{r}\right) & \sqrt{2} a_{j}^{p} \gamma A_{j-3 p / 2}^{(p)} & \gamma_{1} \frac{\partial}{\partial r}+2 f_{-2}^{j, p} \frac{\underline{\chi}}{r}
\end{array}\right)
$$

$$
\begin{gathered}
D_{r}=\frac{\partial}{\partial r}+\frac{3 / 2}{r}, f_{n}^{j, p}=p\left(j+\frac{1}{2}-\frac{n p}{2}\right), \\
\chi=\left(5 \gamma-\gamma_{1}-1\right) / 3
\end{gathered}
$$

The specified boundary conditions allow us to calculate the energy spectrum of spherical heterosystems. If in the formulas (9) - (13) the value of $\Delta$ is directed to infinity, then we obtain the formulas of the 4-band model 
[23], which doesn't take into account the spin-orbital band. heterostructure where spherical GaAs nanocrystal is placed in the AlAs semiconductor matrix. Parameters for semiconductor nanoheterosystem are known [19]:

\section{The hole energy spectrum of the GaAs / AlAs heterostructure}

All numerical calculations have been obtained for

$$
\begin{aligned}
& \text { GaAs }: \gamma_{1}=6.98 ; \gamma_{2}=2.06 ; \gamma_{3}=2.93 ; \gamma=\frac{2 \gamma_{2}+3 \gamma_{3}}{5} ; \Delta=0.341 e B \\
& A l A s: \gamma_{1}^{I I}=3.76 ; \gamma_{2}^{I I}=0.82 ; \gamma_{3}^{I I}=1.42 ; \gamma=\frac{2 \gamma_{2}^{I I}+3 \gamma_{2}^{I I}}{5} ; \Delta^{I I}=0.28 e B ; \quad V_{0}=0.562 e B
\end{aligned}
$$

Analyzing the crystals parameters, it can be seen that the hole and electron states can be calculated separately

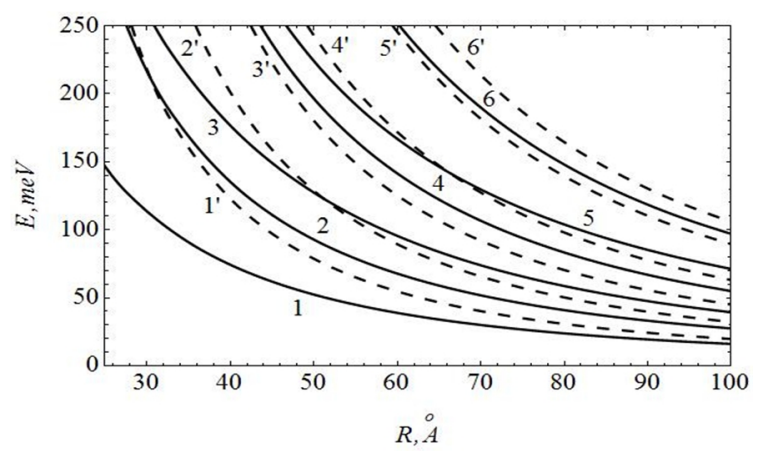

Fig. 1. Dependence of the hole energy on the radius of a quantum dot $R$ : dashed curves are the model of an infinite potential well for a hole; solid curves are the model with a finite gap of bands. Solid curves: even states $1-1 S_{3 / 2}, 2-1 S_{5 / 2}, 4-1 S_{7 / 2}, 5-1 S_{7 / 2}$, odd states $3-1 S_{5 / 2}, 6-1 S_{7 / 2}$; dashed curves: even states $1^{\prime}-1 S_{3 / 2}, 2^{\prime}-1 S_{5 / 2}, 4^{\prime}-1 S_{7 / 2}, 5^{\prime}-1 S_{7 / 2}$, odd states $3^{\prime}-1 \mathrm{~S}_{5 / 2}, 6^{\prime}-1 \mathrm{~S}_{7 / 2}$.

as a result of a rather large difference between the energy of the top of the valence band and the bottom of the conduction band.

That is why, we can use a spherical four-band and six-band Luttinger model for hole states and a oneparabolic parabolic for electron states of a nanoheterosystem.

As can be seen from (16), the values of spin-orbital bands $\Delta, \Delta^{I I}$ are such that they require the use of a sixband model. However, the correct use of models can only be established as a result of numerical calculations. The results of calculations of the energy dependence of the lowest levels on radius $R$ are shown in Fig 1. From the figure it can be seen that for $n_{r} S_{3 / 2} \quad(L=0)$ (S-state) an increase in the radius $R$ is accompanied by a monotonous decrease in the holes energy for each value of the radial quantum number $\left(n_{r}\right)$. If we assume that there is an infinite bands gap $\left(V_{0} \rightarrow \infty\right)$, then the corresponding radial functions (9)-(10), (12)-(13), at the interface $(r=R)$ will be zero. In this case, the dependence $E(R)$ is represented by dashed curves $1^{\prime}-6^{\prime}$. From the results of the comparison of the holes ground state energy $\left(n_{r}=1\right)$ in a finite-gap $\mathrm{V}_{0}$ band model $\left(E_{\mathrm{c}}\right)$ with the model of an infinite $\mathrm{V}_{0}=\infty$ potential well for a hole $\left(E_{\mathrm{b}}\right)$, it turns out that $E_{c}<E_{b}$. These results are fully explained by the Heisenberg uncertainty principle. For numerical data calculations show that for a given heterosystem in the $R>7.5 \mathrm{~nm}$ region, the relative error is $\delta=\frac{E_{b}-E_{c}}{E_{b}} \leq 23.83 \%$.

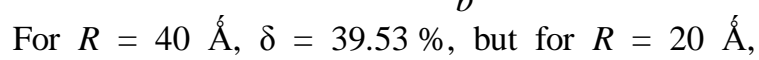
$\delta=59.79 \%$. The energies values of excited states $\left(n_{r}=2,3, \ldots\right)$, calculated in these models, show even greater discrepancy.

Despite the fact that we have obtained a finite number of bound states for $\mathrm{V}_{0}=0.562 e B$, and an infinite number for the infinite potential well model, the relative error for a given $R$ increases the same way as for $n_{r}=1$, that is for $n_{r}=2 R=40 \AA, \eta=45,5 \%$, and for $R=$ $20 \AA \hat{n}, \eta=67,3 \%$.

Fig. 2 shows the dependence of the hole ground state on the QD radius, calculated within the framework of various models. The figure shows the quantitative difference between the results of the six-band, four-band,

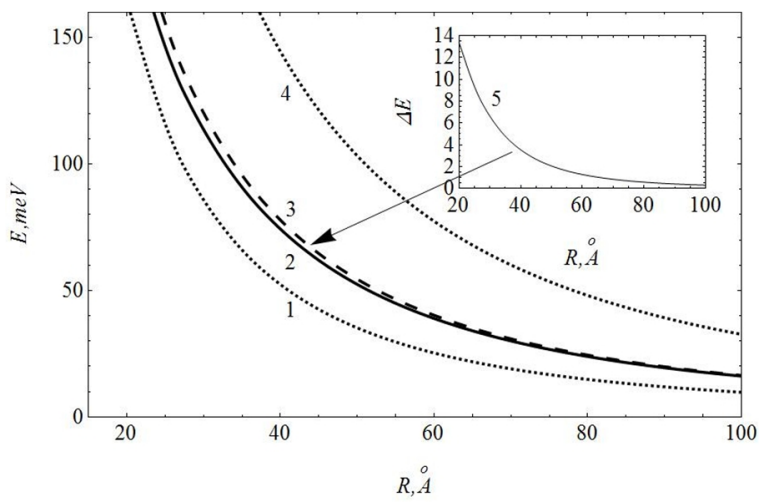

Fig. 2. Energy dependence for the hole ground state, calculated taking into account the four-band approximation (dashed curve 3), six-band approximation (curve 2), the heavy holes band (dashed curve 1), the light holes band (dashed curve 4 ), and the difference $\Delta E$ (insert) between the fourband and a six-band approximation. 
and one-band model of the valence band. Consistent consideration of all formulas will result in the dependence of energy on the radius of QD, which is indicated by curve 2 .

If we use the 4-band approximation (neglected by the spin-orbital band) as in [23] then the energy of the ground state will be described by curve 3 . The interface of the heterostructure has a stronger effect on the energy spectrum due to a decrease in the size of the QD. Therefore, for small radius, the numerical values of energy differ from each other under the specified boundary conditions.

If we neglect the complex structure of the valence band and consider it non-degenerate, and for the calculations to take the mass of a heavy hole, then energy, as a function of the radius of QD, is represented by the dashed curve 1 . The graph shows that less energy is obtained in the one-band model for heavy holes than in the four-band and six-band models. Naturally, the energy of light holes is greater than that of heavy ones. Naturally, the energy of light holes is higher that of heavy holes. In addition, it is larger than the four-band and six-band models.

The sequential account of the spin-split band somewhat reduces the hole energy. As can be seen from the figure (insert) curve 5 at small radius $(R=20 \stackrel{0}{A})$, the four-band approximation (stroke curve 3 ) and sixband approximation (solid curve 2) differ by $13.5 \mathrm{meV}$. And this can affect the absorption spectra in such systems. Increasing the radius of quantum dot to $R=100 \stackrel{0}{A}$ indicates the possibility of using a simpler four-band approximation in this problem.

In the present work the hole states energy of spherical GaAs/AlAs nanoheterostructure in the approximation of the isotropic valence band for the Luttinger Hamiltonian taking into account the final gap of the bands have been defined. In the range of small radius it is necessary to take into account the double degeneration of the hole at the top of the Brillouin band and the spin splitting in the valence band. The states and the finiteness of the spherical quantum well significantly affect the numerical values of the hole energy.

It is possible to calculate the probabilities of interband transitions in the emission and absorption spectra of a given and similar heterostructures on the basis of the formulas obtained for the wave functions of hole states.

The hole states has been calculated using four-band and six-band models for spherical QD. It is shown that, neglecting the complex structure of the valence band, obtained or understated (consider only to the heavy holes band) or inflated (consider only to the light holes band) energy compared to multi-zone models. This will allow us to obtain the energies of the acceptor states for arbitrary radius and to agree with the results of experimental work in massive crystals. It is also shown that for small radius of QDs $(2-4 \mathrm{~nm})$ in crystals with a large bandgap and an intermediate value of the spinorbital band, a complete 6-band Hamiltonian should be used for hole states. For larger radius, the 6-band model can be approximated by 4-band model.

Bilynskyi I. - Professor, Doctor of Physical and Mathematical Sciences;

Leshko R. - Candidate of Physical and Mathematical Sciences, docent of Physics Department;

Metsan H. - Master's Degree Student of Physics Department;

Shevchuk I. - Lecturer of Physics Department.

[1] V. Sivasubramanian, A.K. Arora, M. Premila, C.S. Sundar, V.S. Sastry, Physica E 31(1), 93 (2006). (http://dx.doi.org/10.1016/j.physe.2005.10.001).

[2] P. Ardalan, T.P. Brennan, H.-B.-R. Lee, J.R. Bakke, I-K. Ding, M.D. McGehee,S.F. Bent, ACS Nano 5(2), 1495 (2011) (http://dx.doi.org/10.1021/nn103371v).

[3] K.-L. Chou, H. Meng, Y. Cen, L. Li, J.-Y. Chen, J. Nanopart. Res. 15, 1348 (2012) (http://dx.doi.org/10.1007/s11051-012-1348-9).

[4] L. Shao, Y. Gao, F. Yan, Semiconductor quantum dots for biomedical applications, Sensors 11, 11736 (12) 11736-11751. (http://dx.doi.org/10.3390/s111211736).

[5] T. Pons, H. Mattoussi, Ann. Biomed. Eng. 37(10),1934 (2009) (http://dx.doi.org/10.1007/s10439-009-9715-0).

[6] J.C.C. Santos, A.A.P. Mansur, H.S. Mansur, Molecules 18(6), 6550 (2013) (http://dx.doi.org/10.3390/molecules18066550).

[7] A.I. Ekimov, I.A. Kudryavtsev, M.G. Ivanov, Al.L. Efros, J. Lumin. 46(2), 83 (1990). (http://dx.doi.org/10.1016/0022-2313(90)90010-9).

[8] N.A. Efremov, S.I. Pokutnii, Solid State Phys. 32(10), 2921 (1990).

[9] S.I. Pokutnii, Semiconductors 31(12), 1247 (1997). (http://dx.doi.org/10.1134/1.1187305).

[10] V.I. Klimov, J. Phys. Chem. B 104 (26), 6112 (2000) (http://dx.doi.org/10.1021/jp9944132).

[11] N.V. Tkach, Yu.A. Sety, $\quad$ Semiconductors 1083 (http://dx.doi.org/10.1134/S106378260609017X).

[12] N.V. Tkach, Yu.A. Seti, Phys. Solid State 51(5), 1033 (http://dx.doi.org/10.1134/S1063783409050230).

[13] M. Bissiri, G. Baldassari H.v.H., M. Capizzi,V.M. Fomin, V.N. Gladilin and J.T. Vreese, Phys.status solidi (b) 224(3), $639 \quad$ (2001). $\quad$ (https://doi.org/10.1002/(SICI)1521-3951(200104)224:3<639::AID-PSSB639> 3.0.CO;2-1). 
[14] V.I. Boichuk, I.V. Bilynskyi, I.O. Shakleina, V.B. Holskyi, Condensed Matter Physics 13(1), 1370 (2010). (10.5488/CMP.13.13701).

[15] V.I. Boichuk, I.V. Bilynskyi, R.Ya. Leshko, L.M. Turyanska, Physica E: Low-dimensional Systems and Nanostructures 44, 476 (2011) (10.1016/j.physe.2011.09.025).

[16] [16] V.I. Boichuk, R.Ya. Leshko, I.V. Bilynskyi, L.M. Turyanska, Condensed Matter Physics, 2012, vol. 15, No. 3, 33702:1-10 (https://doi.org/10.5488/CMP.15.33702 )

[17] V.I. Boichuk, I.V. Bilynskyi, R.Ya. Leshko, L.M. Turyanska, Physica E: Low-dimensional Systems and Nanostructures 54, 281 (2013) (10.1016/j.physe.2013.07.003).

[18] C. Hermann, C. Weisbuch, Phys. Rev. B 15(2), 823 (1977) (http://dx.doi.org/10.1103/PhysRevB.15.823).

[19] A. Baldereschi, N.O. Lipary, Phys. Rev. B 8(6), 2697 (1973) (http://dx.doi.org/10.1103/PhysRevB.8.2697).

[20] G.B. Grigoryan, E.M. Kazaryan, Al.L. Efros, T.B. Yazeva, Solid State Phys. 32(6), 1722 (1990) (Russian).

[21] В.I. Шека, Д.І. Шека, ЖЕТФ 51(5), 1445 (1967).

[22] D.J. Ben Daniel and C.B. Duke, Phys. Rev. 152, 683 (1966).

[23] V.I. Boichuk, I.V. Bilynskyi, R.Ya. Leshko, I.O. Shakleyina, Ukrainian Journal of Physics 55(3), 326 (2010).

\author{
I.В. Білинський, Р.Я. Лешко, Х.О. Мецан, І.С. Шевчук
}

\title{
Діркові стани в сферичних квантових наногетероситемах 3 проміжною спін-орбітальною взаємодією
}

\author{
Дрогобиџький державний педагогічний університет імені Івана Франка, 82100 Дрогобич, Украйна, \\ e-mail: leshkoroman@gmail.com
}

\footnotetext{
Спектр енергії дірок вивчений для сферичної напівпровідникової наногетеросистеми 3 кубічною симетрією. Точні рішення рівняння Шредінгера для основного та збудженого станів дірок представлені в рамках 6-смугового гамільтоніана Луттінгера та кінцевого проміжку смуг 3 відповідними граничними умовами. Для гетероструктури GaAs / AlAs обчислено залежність енергій дірок від радіуса квантової точки. Отримані результати порівняно 3 даними, отриманими за допомогою моделі нескінченного потенційного колодязя, а також однодіапазонної моделі для важких і легких дірок.

Ключові слова: квантовий дон, спектр дірок, 6-діапазонна модель.
} 